\title{
Enhancement of interfacial fracture toughness of carbon/epoxy composite adhesive joints by in-mold surface preparation
}

\author{
Y. Hikosaka ${ }^{1}$, R. Matsuzaki2* ${ }^{*}$ A. Todoroki ${ }^{1}$, Y. Mizutani $^{1}$ \\ ${ }^{1}$ Department of Mechanical Sciences and Engineering, Tokyo Institute of Technology, 2-12-1-I1-58, O-okayama, Meguro, \\ 152-8552 Tokyo, Japan \\ ${ }^{2}$ Department of Mechanical Engineering, Tokyo University of Science, 2641 Yamazaki, Noda, 278-8510 Chiba, Japan
}

Received 16 September 2012; accepted in revised form 19 November 2012

\begin{abstract}
To reduce secondary processing such as sanding or chemical etching in the manufacture of composite structures, we present an in-mold surface preparation process using imprint lithography on carbon/epoxy composite adhesive joints. In the proposed in-mold process, microstructures are designed and fabricated on the surface of the mold to form composites. Through the formation of composites on the mold at an appropriate temperature and pressure, the shapes of the microstructures are imprinted onto the surface of the composite. Because molding and surface preparation can be performed simultaneously, the time and costs required are reduced compared to conventional surface preparations. In this paper, concavo-convex microstructures were fabricated on the surface of carbon/epoxy composites using in-mold surface preparation, which improved the apparent mode I fracture toughness of the composite/adhesive interface. From double-cantilever-beam tests, we confirmed that as the aspect ratio of the concavo-convex microstructures increased, the steady-state fracture toughness increased by up to $113 \%$ compared to structures without in-mold surface preparation, and the fracture mode changed from interfacial failure to complex cohesive adhesive failure.
\end{abstract}

Keywords: polymer composites, processing technologies, adhesion, in-mold surface preparation, imprint lithography

\section{Introduction}

The use of composite materials such as carbon-fiber reinforced plastics (CFRPs) within the automotive industry is increasing. In these mass-production industries, composite structures need to be efficiently manufactured [1]. The efficiency with which composite structures can be produced depends on the molding process and secondary fabrication processes such as trimming or surface preparation. Short-cycle resin transfer molding (RTM), which enables the formation of CFRPs in only $10 \mathrm{~min}$, has been developed for the mass production of CFRP parts [2]. This short-cycle RTM was made possible by the development of rapid-cure resin and the rapid-impregnation method.

Secondary fabrication processes that involve conventional surface preparations such as sanding, sand blasting, plasma treatment, or chemical etching, are too time consuming to be applicable to mass production, especially for the production of largescale structures. Furthermore, workers who are not properly protected may be exposed to carbon particulates suspended in the air or to the harmful chemicals used in surface-preparation processes [3]. To improve the production of CFRP structures, it is essential to reduce the number of secondary fabrication process.

\footnotetext{
${ }^{*}$ Corresponding author, e-mail: rmatsuza@rs.tus.ac.jp

(C) BME-PT
} 
Thus, we have proposed an in-mold surface preparation process using imprint lithography to produce bonded butt joints [4]. Imprint lithography was first proposed as nanoimprint lithography (NIL) by Chou and coworkers [5-6]. In this process, nanometer-scale structures on a mold are pressed onto melted polymeric material at appropriate temperature and pressure and the shape of nanostructures is transferred to the surface of the polymer. Because the nanostructures can be easily fabricated at low cost and with high throughput and resolution, the process has been applied in the semiconductor device industry to, for example, produce nanometer-scale metal-oxide-semiconductor field-effect transistors [7], a nanofluidic chip for DNA stretching [8], and optofluidics for environmental monitoring applications [9]. In our previous study [4], we performed the in-mold surface preparation of composite materials with imprint lithography using a silicon wafer as a mold; the surface of the wafer was photo-lithographically fabricated with the designed microstructures. This imprint technique can be used to imprint the shape of microstructures onto the surface and obtain an adherent surface for adhesive joining without the need for harmful sanding or sand blasting. Because the formation of composites and surface preparation can be performed simultaneously, this technique is faster and less expensive than conventional surface treatments. We fabricated pyramidal microstructures using in-mold surface preparation and experimentally determined the effect of the process on the tensile bonding strength of butt joints. The tensile strength of butt joints was found to be $67 \%$ higher than that of untreated joints. Although it has been reported that interfacial properties such as interfacial strength and fracture toughness are highly dependent on the surface topography of adherends, the effect of the shape and size of the microstructures created by in-mold surface preparation on the joint strength has not been studied [1019]. Moreover, the resistance to crack propagation at the imprinted interface has not been investigated, although the fracture of bonded joints is likely to be caused by crack initiation and propagation at this interface.

Therefore, the present study investigated the effect of the shape of imprinted microstructures on the steady-state mode I fracture toughness of the CFRP/ adhesive interface, with the goal of controlling the interfacial properties of composites by appropriately designing and fabricating microstructures on the mold surface. The concavo-convex microstructures were fabricated on the CFRP surface and the effect of the shape of the microstructures on the mode I fracture toughness was investigated using double-cantilever-beam (DCB) tests. Crack propagation behavior was also investigated with in situ microscopic observations.

\section{In-mold surface preparation using imprint lithography}

\subsection{Concavo-convex microstructures}

The driving forces of two-dimensional (2D) crack propagation are divided into mode I forces, which open a crack perpendicular to the crack surface, and mode II forces, which open the crack parallel to the surface. Generally, the fracture toughness is a measure of not only the energy required for surface generation but also the energy required for plastic deformation or to overcome friction during crack growth. The latter energy is called dissipation energy, and the dissipation energy of mode II is usually higher than that of mode I [20]. From the above observation, a concavo-convex shape, as shown in Figure 1, was selected for microstructures to improve the apparent mode I fracture toughness $G_{\mathrm{IA}}$ of an adhesive joint; $G_{\mathrm{IA}}$ indicates the resistance to macroscopic crack propagation when the macroscopic mode I load is applied to the CFRP/adhesive interface. In this calculation of the apparent fracture toughness, the concavo-convex microstructures are assumed to be a flat interface. It is also noted that this $G_{\text {IA }}$ contains a microscopic mode II component as well as a microscopic mode I component. Here, the shape of concavo-convex microstructures is characterized by the aspect ratio given by Equation (1):
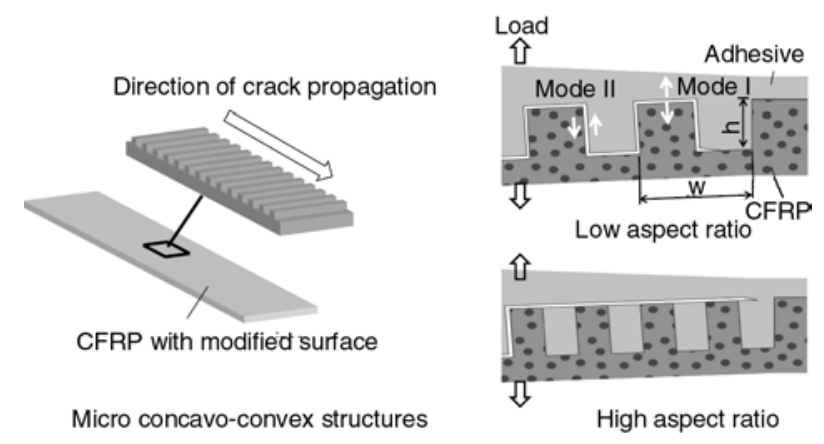

Figure 1. Schematic of concavo-convex microstructures and the fracture mode transition as the aspect ratio $A$ is changed 
$A=\frac{h}{w}$

where $w$ is the summation of the widths of one convexity and one concavity and $h$ is the height (see Figure 1). Note that $A=0$ indicates a flat surface without concavo-convex microstructures.

Because the fracture toughness of cohesive failure is usually higher than that of interfacial failure [16], a crack may propagate along the interface of concave-convex microstructures. In this fracture mode, the actual length of microscopic cracks increases near concave-convex microstructures and mode II interfacial failure also occurs at the lateral faces of the microstructures. Because the mode II fracture toughness is usually higher than that of mode I in practical adhesion, higher energy is required for crack propagation. Thus, the crack resistance may increase as a result. As the aspect ratio of the concavo-convex microstructures becomes high enough, as shown in Figure 1, cohesive failure inside the adhesive or CFRP may occur, accompanied by interfacial failure. The fracture toughness of this fracture mode is not affected by the interfacial property but is influenced by the fracture toughness of the adhesive or of CFRP; thus, this mode is preferable for the realization of stable bonding. Because the ratio of the mode II fracture region increases in proportion to the aspect ratio of the concavo-convex microstructures, we evaluated the fracture toughness by changing this ratio.

\subsection{Process of in-mold surface preparation}

Concavo-convex microstructures were fabricated on the CFRP surface by in-mold surface preparation. This surface preparation follows the NIL procedure, which is a pattern-transferring technique in which microstructures of a mold are pressed into low-viscosity plastics at high temperature and patterns are transferred by demolding at low temperature. The technique was introduced during the curing of composites. Figure 2 shows a schematic of the application process to the adhesion layer between the CFRP panel and the stiffener. The steps in this process are as follows:

(1) Concavo-convex microstructures were fabricated on an aluminum plate using a milling machine. After coating the mold surface with releasing agent (ChemTrend, Chemlease \#70), carbon/epoxy prepregs (Mitsubishi rayon, Pyro-
(1) Stacking

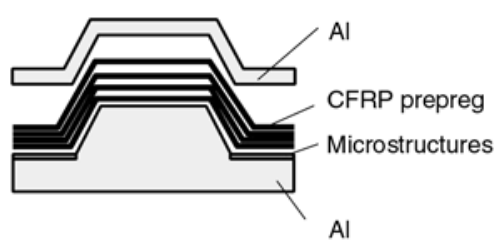

(2) Curing

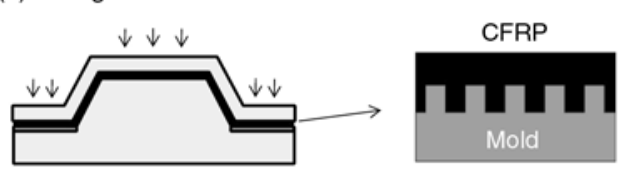

(3) Demolding

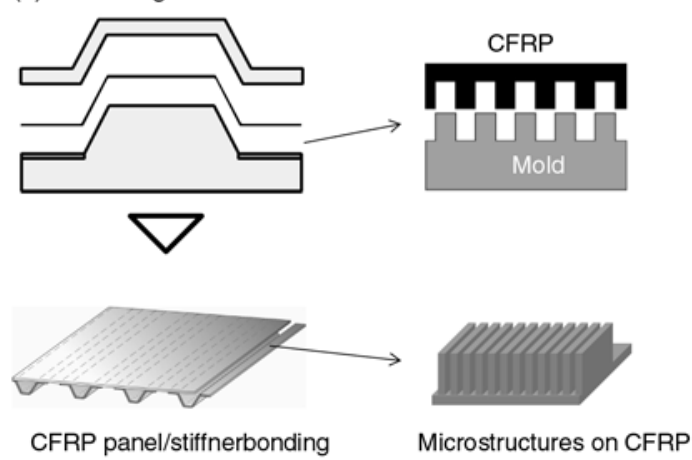

Figure 2. Process of in-mold surface preparation and application to the adhesive surface of a carbon-fiber reinforced plastic (CFRP) panel and stiffener

fil \#380) were stacked on the mold. The stacking sequence was $[90 / \pm 45 / 0]_{S}$ in this study, with the concave and convex directions set to $90^{\circ}$; thus, the fiber direction in the top layer must be identical to the convex direction.

(2) Prepregs were cured in two steps over the glass transition temperature under a pressure of $0.6 \mathrm{MPa}\left(85^{\circ} \mathrm{C}\right.$ for $2 \mathrm{~h}$ and $135^{\circ} \mathrm{C}$ for $\left.3.5 \mathrm{~h}\right)$, which allowed, the melted matrix resin to flow into the microstructures of the mold.

(3) Microstructures were transferred to the CFRP by demolding at room temperature.

Figure 3 shows a schematic and a photograph of the mold. In the present study, microstructures of several sizes were fabricated on an aluminum plate. This enabled us to simultaneously fabricate CFRP laminates with microstructures of several aspect ratios, which increased the efficiency with which specimens were prepared for the experiments described later.

Figure 4 shows images of the surface of the imprinted CFRP taken with a scanning electron microscope (SEM; Keyence, VE-8800). In this study, five micro- 

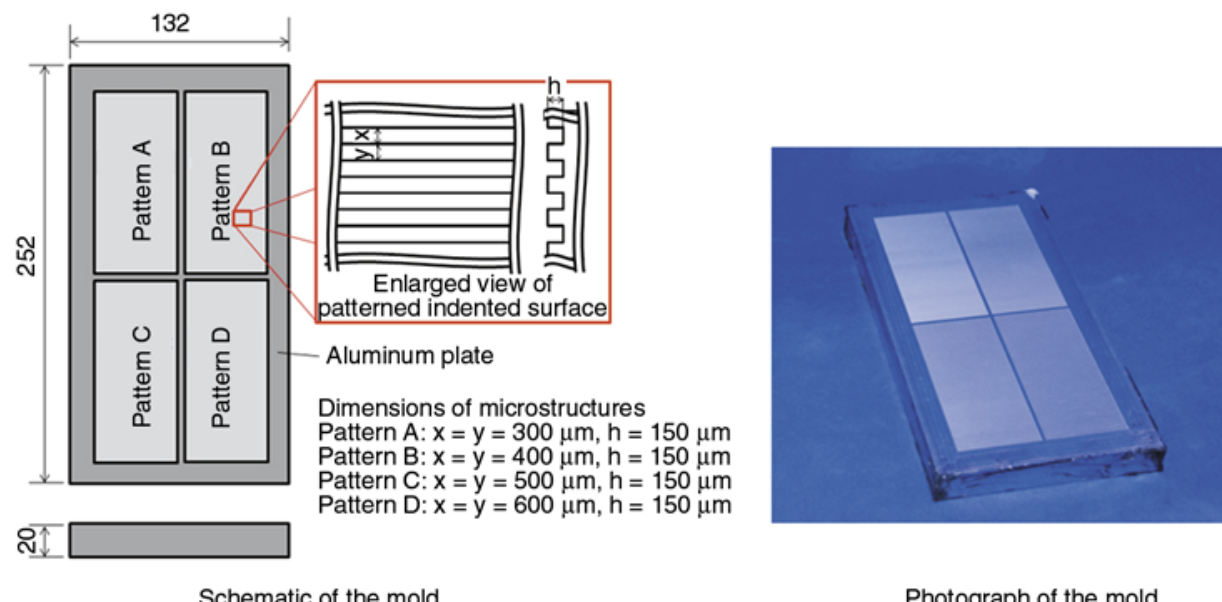

Photograph of the mold

Figure 3. Schematic and photograph of the mold with microstructures

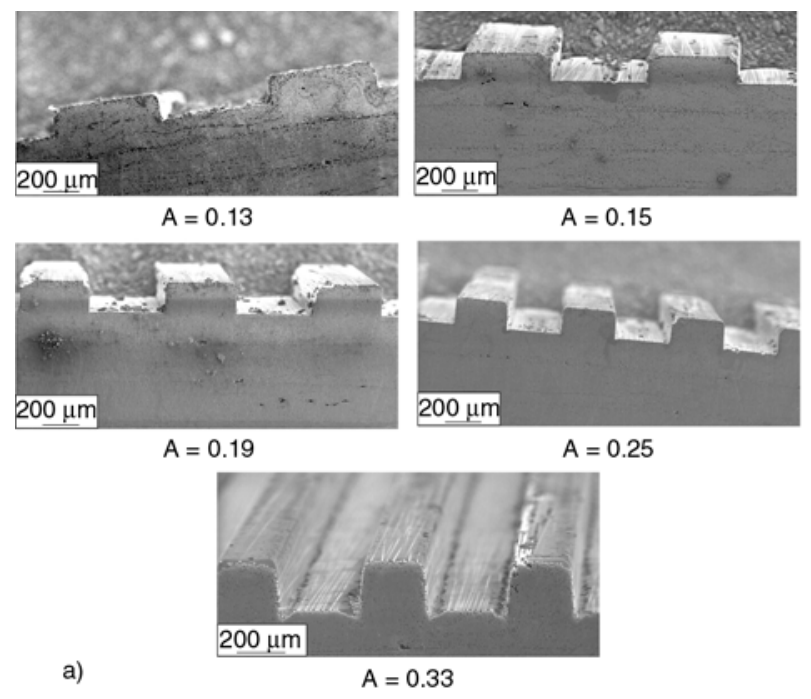

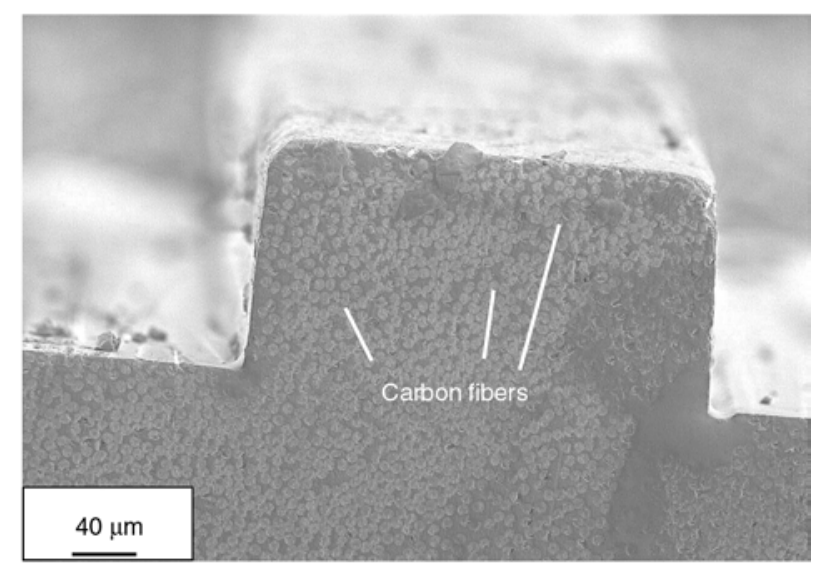

b)

Figure 4. Scanning electron microscope images of microstructures on the surface of CFRPs treated by in-mold surface preparation: (a) images of the microstructures on CFRPs; (b) image of the enlarged view of a microstructure $(A=0.25)$

structure shapes were fabricated on the CFRP using aspect ratios $(A)$ of $0.13,0.15,0.19,0.25$, and 0.33 . At aspect ratios ranging from 0.13 to 0.25 , the height was set at a constant value of $150 \mu \mathrm{m}$ as the width was changed to $600,500,400$, and $300 \mu \mathrm{m}$; for $A=0.33$, the height was $200 \mu \mathrm{m}$ and the width was $300 \mu \mathrm{m}$ because the milling machine can create a line no narrower than $300 \mu \mathrm{m}$. From the images in Figure 4, we confirmed that all of the microstructure shapes were successfully transferred to the CFRP surface. The convex microstructures were observed to contain a number of carbon fibers, and thus behave as composites at this scale.

\section{Experimental procedures 3.1. DCB specimen}

We conducted DCB tests to investigate the resistance to crack propagation along the CFRP/adhesive interface under macroscopic mode I loading. The
DCB testing and data processing follow Japan Industrial Standards (JIS) K 7086, the testing method used to evaluate the interlaminar fracture toughness of CFRPs [21]. Figure 5 shows the DCB specimen configuration. After cutting the imprinted CFRP $[90 / \pm 45 / 0]_{S}$ into specimens $100 \mathrm{~mm}$ in length and $15 \mathrm{~mm}$ in width, the CFRP was cleaned off with a surface-active agent (Kao, Kitchen Haiter). Note that the specimen size is somewhat smaller than the size prescribed in the JIS protocol, due to limitations in fabricating the imprinted area. The proposed in-mold surface preparation was applied to one adherend, and the surface of the other adherend was treated using emery paper $\# 240$. In the range of the tested aspect ratio, we confirmed the crack propagates between the adhesive and in-mold prepared surface. The fracture toughness of the adherend treated with emery paper and that of the adherend treated using the proposed method are compared in 


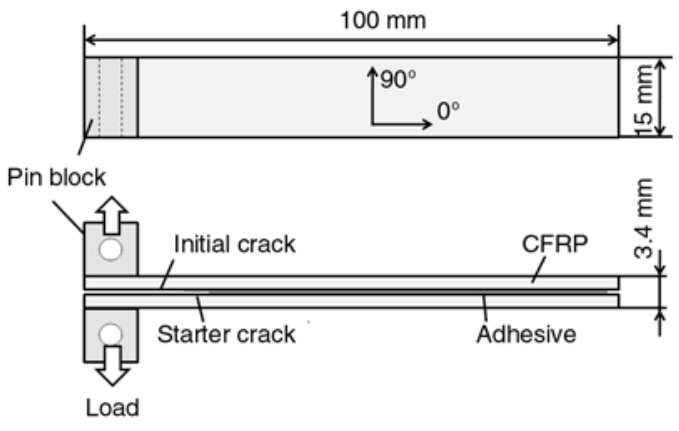

Figure 5. Configuration of a double-cantilever-beam (DCB) specimen

Section 4.2. Two CFRP adherends were bonded together using an epoxy adhesive (3M, DP-105) under pressure (about $0.01 \mathrm{MPa}$ ) using two aluminum plates as well as a plummet for $48 \mathrm{~h}$ at room temperature, with Teflon sheets $(0.1 \mathrm{~mm}$ thickness) inserted between two adherends at both edges to introduce an initial crack and to control the thickness of the adhesive layer. Thus, the thickness of the adhesive layer was $0.1 \mathrm{~mm}$, and the thickness of the fabricated DCB specimen was about $3.4 \mathrm{~mm}$. After bonding, the excessive adhesive flowing out of the specimen was removed using emery paper. Before tests were conducted, a starter crack was fabricated by inserting a cutter knife. To evaluate the effect of the shape of the microstructures on the resistance to crack propagation and crack-propagation behavior, six kinds of adherends including a flat surface were prepared: $A=0,0.13,0.15,0.19,0.25$, and 0.33 . For each aspect ratio, three specimens were tested.

\subsection{DCB tests for mode I fracture toughness}

DCB tests were performed using a tensile testing machine (Shimadzu, AG-I) under crack opening displacement (COD) control at $0.2 \mathrm{~mm} / \mathrm{min}$. The $\mathrm{COD}$ is defined as the relative displacement between two attached pin blocks. During DCB tests, load, crack length, and COD as a function of time were recorded. The crack-propagation behavior at the CFRP/adhesive interface was observed using a digital microscope (Scholar, M3), and the crack length was measured with a measuring microscope (Pikaseiko, PRM-2). After the crack had propagated sufficiently (forming a crack about $25 \mathrm{~mm}$ long), the specimen was fully unloaded and the permanent offset displacement, $\delta_{\text {offset, }}$ was measured to check the quantity of plastic deformation. Our procedure to calculate fracture toughness is discussed in the next section.

\section{Results and discussion \\ 4.1. Evaluation of crack-propagation resistance}

Because the proposed surface preparation produces a concavo-convex bonding interface, the crack-propagation resistance varies microscopically depending on the crack position within the concavo-convex shape. However, the objective of this study was not to obtain the precise crack-propagation resistance on a microscopic scale but to evaluate the macroscopic crack-propagation resistance, as a practical indicator of the effect of using the proposed surface preparation instead of conventional treatments. Therefore, we first investigated the method used to examine this resistance.

Figure 6 shows representative load-COD curves of the DCB tests for various aspect ratios ranging from 0 to 0.33 . For all specimens, the load increases linearly up to the critical value to propagate the crack; the load then decreases smoothly as the crack length increases, although concavo-convex microstructures exist along the crack path. The observed $\delta_{\text {offset }}$ was within the $10 \%$ of the maximum COD suggested in JIS, implying that no permanent deformation of the adherend had occurred. It is normal for small final offsets to be observed because of the presence of the deformed adhesive slightly propping open the crack [22]. Note that the low gradient of the initial slope of $A=0.33$ is the result of the slightly longer initial crack length fabricated manually by the cutter knife, which increases compliance. It is noted that we did not implement any specific control process for fabricating a constant and fine starter crack for simplicity. Thus, we use the crack propagating resistance when the crack stably propagates for some length in instead of using the initial crack propagating resistance for evaluation.

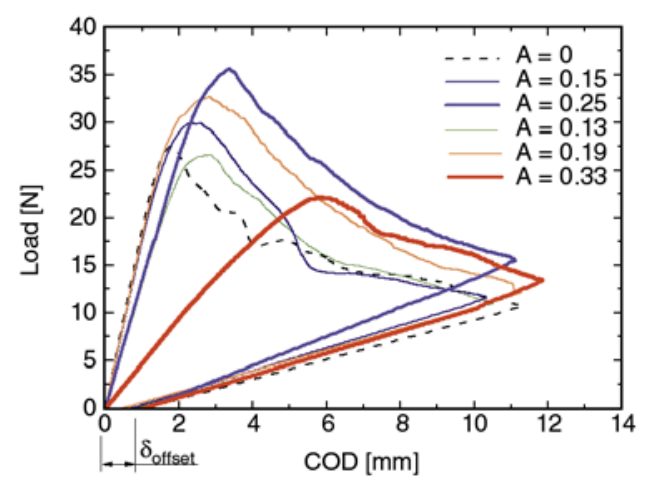

Figure 6. Representative load-crack opening displacement curves of DCB tests for various aspect ratios 


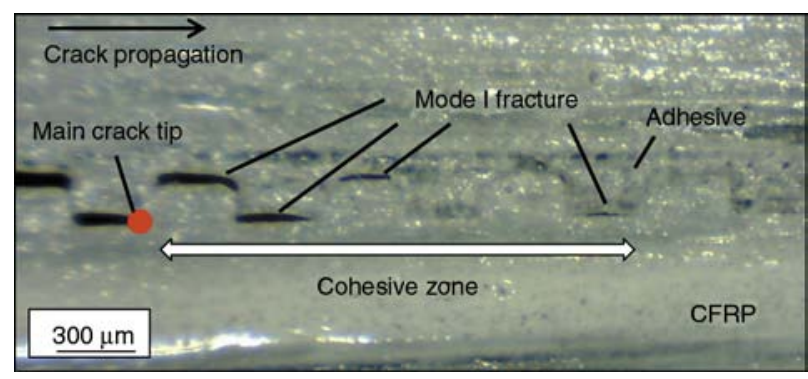

Figure 7. Microscopic image of cohesive zone ahead of the main crack tip during DCB tests

Figure 7 shows the microscopic image around the crack tip obtained during DCB tests of the $A=0.25$ specimen, and shows the formation of multiple microscopic cracks ahead of the macroscopic main crack tip (the red circle in Figure 7), which penetrated from the left edge. We refer to this zone of multiple micro-cracks, ahead of the main crack, as the cohesive zone in this study; in this region, a cohesive force exists in the closure direction of crack surfaces $[23,24]$. In this cohesive zone, mode I fracture occurred at the horizontal interfaces, whereas the lateral interfaces remained bonded. This was attributed to the fact that the fracture toughness of mode I is lower than that of mode II. This crackpropagation behavior was observed for all specimens, with the exception of the flat specimen, $A=$ 0 . It is also noted that the crack does not propagate

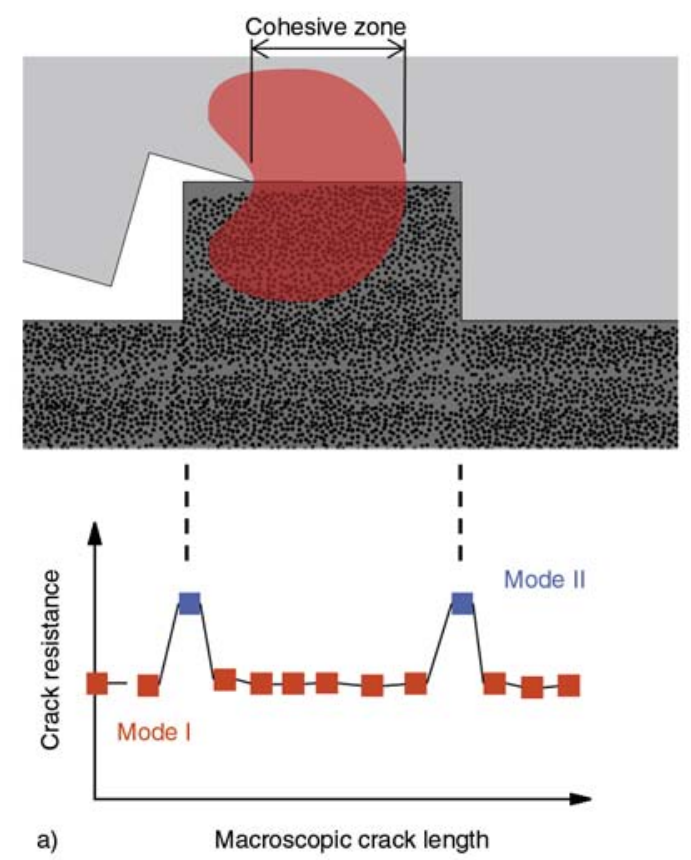

along a straight interface rather than the concavoconvex interface because the apparent fracture toughness of the $A=0.25$ surface was lower than that treated using emery paper \#240 (as mentioned in Section 4.2).

When the size of the concavo-convex microstructure is sufficiently larger than that of the cohesive zone, the crack-propagation resistance may vary depending on the crack-tip position, as shown in Figure 8a. However, if multiple concavo-convex microstructures are included in the cohesive zone, as in the present study, energy dissipates more uniformly owing to the presence of the multiple microscopic cracks that allow the propagation of cracks that are independent of the main crack position in the concavo-convex microstructures, and the crackpropagation resistance has a more uniform value (Figure 8b). In the DCB experiments, the load decreased smoothly after crack propagation and variation in microscopic load due to the presence of the microstructures, such as stick-and-slip behavior, did not occur. Therefore, the uniform crack-propagation resistance of the concavo-convex microstructures was evaluated using the 'apparent fracture toughness' by assuming a flat interface. The apparent fracture toughness corresponds to the total energy dissipation including the cohesive zone, divided by the macroscopic crack length.
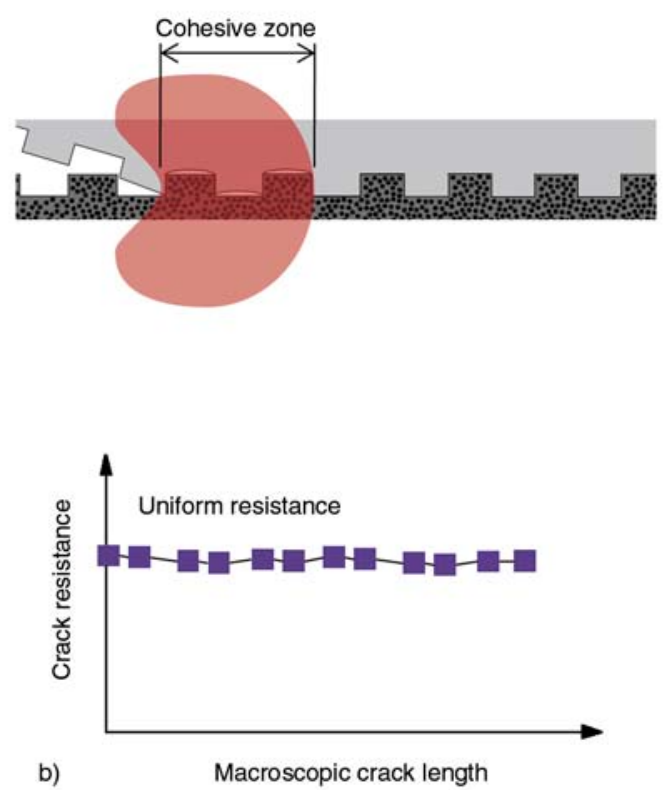

Figure 8. Schematics of cohesive zone ahead of the main crack tip and effects of crack-tip position on crack resistance: (a) large-scale structures (on the order of millimeters); (b) microstructures (on the order of micrometers) 
The apparent mode I fracture toughness $G_{\text {IA }}$ was calculated by Equation (2) in the same manner as JIS K 7086 [21]:

$G_{\mathrm{IA}}=\frac{3}{2(2 H)}\left(\frac{P}{B}\right)^{2} \frac{(B \lambda)^{\frac{2}{3}}}{\alpha_{1}}$

where $2 H$ and $B$ are the thickness and width of the specimen, respectively; $P$ is the applied load; and $\lambda$ is the compliance of the specimen, calculated according to Eqution (3):

$\lambda=\frac{\delta}{P}$

where $\delta$ is COD. $\alpha_{1}$ is a coefficient associated with the flexural rigidity of the adherend, which is obtained by least-squares approximation from the following Equation (4):

$\frac{a}{2 H}=\alpha_{1}(B H)^{\frac{1}{3}}+\alpha_{0}$

where $a$ is the macroscopic clack length, which corresponds to the distance between the crack tip and loading point in the longitudinal direction of the specimen. It should be noted that because the permanent offset displacement $\delta$ offset for all specimens shown in Figure 6 was less than $10 \%$ of the maximum COD, the effects of plastic deformation can be ignored [21].

\subsection{Apparent fracture toughness and crack propagation behavior}

Figure 9 shows a representative $R$ curve indicating the apparent mode I fracture toughness $G_{\text {IA }}$ as a function of the crack length. Although $G_{\text {IA }}$ was unstable during the initial stage of crack propaga-

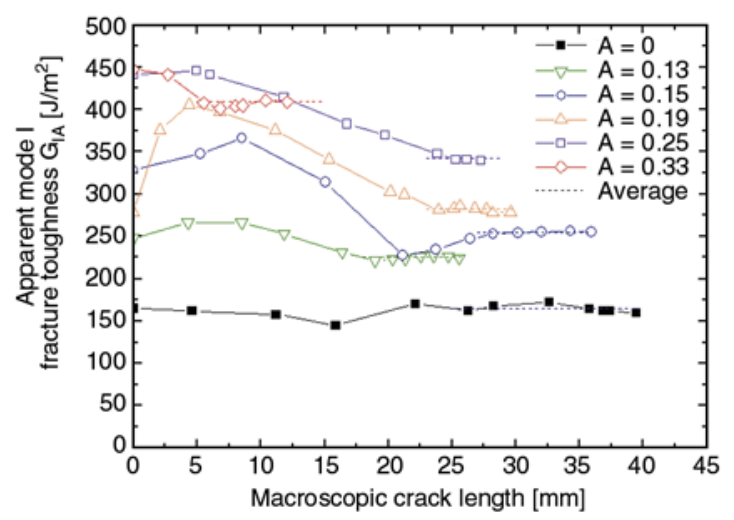

Figure 9. Representative relationships between the apparent mode I fracture toughness $G_{\mathrm{IA}}$ and crack length for various aspect ratios

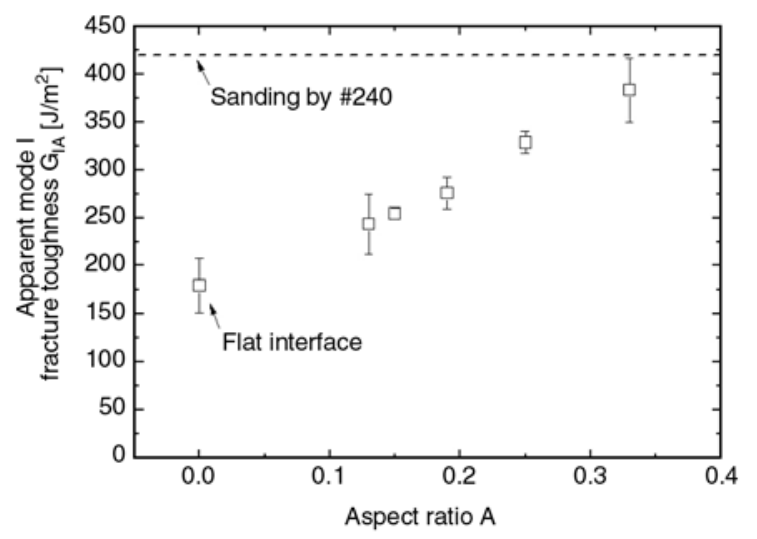

Figure 10. Apparent mode I fracture toughness $G_{\text {IA }}$ as a function of $A$. The dashed line indicates $G_{\mathrm{IA}}$ of a specimen treated by conventional sanding treatment

tion, it became constant as crack propagation progressed. The initial instability was caused by the unstable configuration of the starter crack fabricated by a cutter knife. The dashed line shows the steady-state fracture toughness, which was obtained by averaging the five points.

Figure 10 shows the steady-state $G_{\mathrm{IA}}$ as a function of the aspect ratio $A$. It is noted that the $G_{\mathrm{IA}}$ is apparent macroscopic mode I fracture toughness including the effect of the presence of a microscopic mode II component. It can be seen from Figure 10 that the $G_{\mathrm{IA}}$ after in-mold surface preparation was enhanced compared to that of specimens with a flat interface. Furthermore, $G_{\mathrm{IA}}$ became higher as the aspect ratio $A$ increased and improved by up to $113 \%$ at $A=0.33$ compared to samples without in-mold surface preparation. The dashed line shows the mode I fracture toughness of the composite bonded joint treated by a conventional sanding surface preparation using \#240 emery paper [25]. Table 1 lists the average and standard deviation of $G_{\mathrm{IA}}$ of inmold preparations $(A=0.33)$ and sanding preparations (\#240). Although the average $G_{\text {IA }}$ of the inmold preparations is somewhat lower $(91 \%)$ than that of the sanded preparation, the in-mold preparation achieves almost the same effect as sanding

Table 1. Apparent mode I fracture toughness $G_{\text {IA }}$ of composite bonded joints treated by in-mold surface preparation and by conventional sanding treatment

\begin{tabular}{|l|c|c|}
\hline & $\begin{array}{c}\text { In-mold surface } \\
\text { preparation } \\
(\mathbf{A}=\mathbf{0 . 3 3})\end{array}$ & Sanding by \#240 \\
\hline$G_{\mathrm{IA}}\left[\mathrm{J} / \mathrm{m}^{2}\right]$ & 383 & 419 \\
\hline $\mathrm{SD}\left[\mathrm{J} / \mathrm{m}^{2}\right]$ & 33 & 49 \\
\hline$G_{\mathrm{IA}} / G_{\mathrm{IA}(\mathrm{A}=0)}$ & 2.13 & 2.33 \\
\hline
\end{tabular}




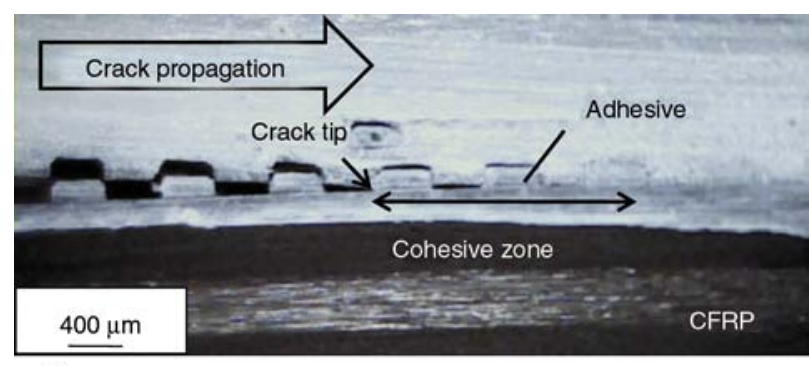

a)

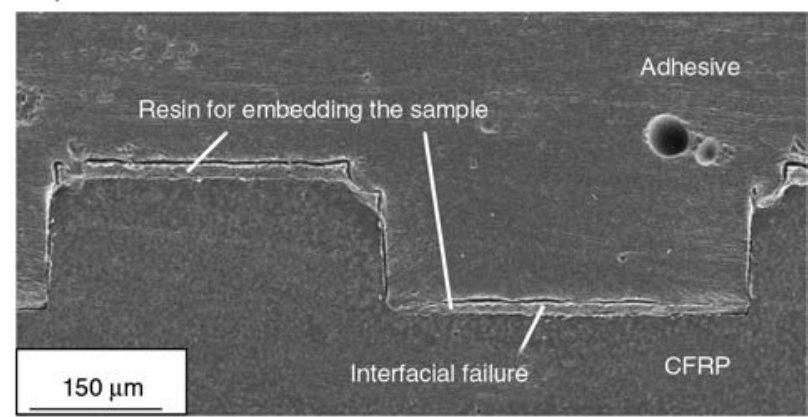

b)

Figure 11. Microscopic images of CFRP/adhesive interfaces $(A=0.19)$ : (a) digital microscope image during the DCB tests; (b) field-emission scanning electron microscope image after the DCB tests

(91\%), but without the need for adverse secondary processes, which enables fast and cost-effective mass production.

Figures 11a and 11b show microscopic images of the CFRP/adhesive interface with $A=0.19$, obtained using a digital microscope (Scholar, M3) during DCB tests and with a field-emission SEM (FESEM; Hitachi, s4500) after the DCB tests, respectively. The microscopic images show that the crack propagates along the interface of the concavo-convex microstructures whereas no crack was observed within either the adhesive or the CFRP (no cohesive failure). The specimens with $A<0.19$ show the same interfacial failure mode along the concavo-convex interface. This fracture behavior implies that as the aspect ratio $\mathrm{A}$ increases (up to $A=0.19$ ), the mode II interfacial failure area per unit length in a specimen's longitudinal direction increases; this is attributed to the increase in the apparent fracture toughness.

Figures 12 and 13 show microscopic images of the failure interface in the $A=0.25$ and 0.33 specimens, respectively. As shown in Figure 12, cohesive failure of the adhesive was observed near the corners of microstructures at the aspect ratio $A=0.25$, in addition to interfacial failure. A comparison of Figure 13 with Figure 12 shows that cohesive failure

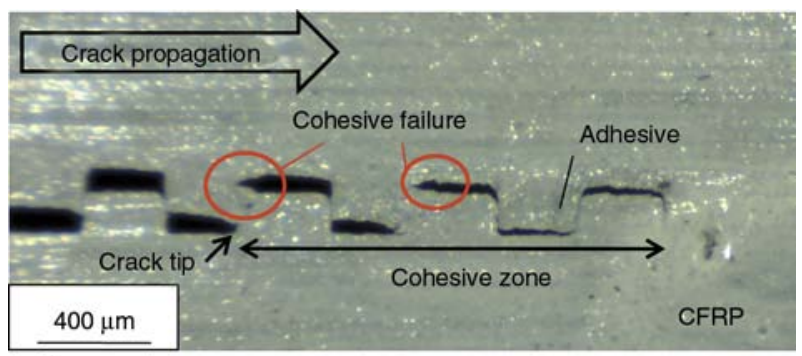

a)

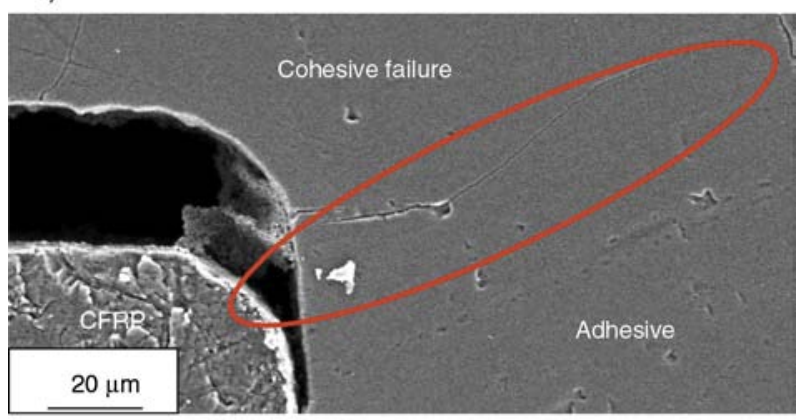

b)

Figure 12. Microscopic images of CFRP/adhesive interfaces $(A=0.25)$ : (a) digital microscope image during the DCB tests; (b) FE-SEM image after the DCB tests
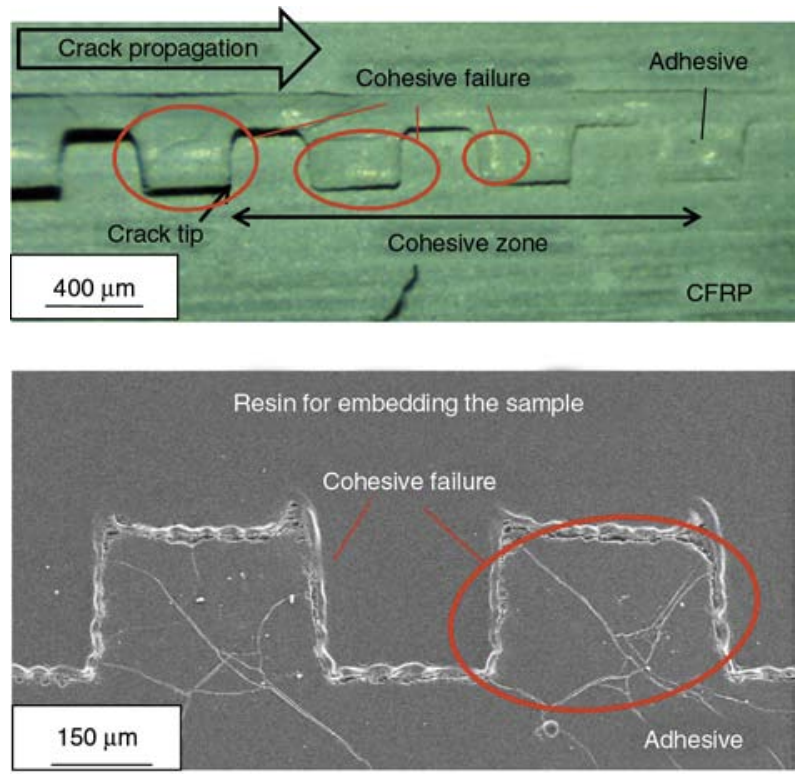

Figure 13. Microscopic images of CFRP/adhesive interfaces $(A=0.33)$ : (a) digital microscope image during the DCB tests; (b) FE-SEM image after the DCB tests

was more complex at $A=0.33$. This agrees qualitatively with the expectation, shown in Figure 1, that interfacial failure is dominant at a low aspect ratio, whereas cohesive failure occurs and enlarges as A increases, although the cohesive fracture mode is more complex. This is attributed to the complex stress distribution at the convex corner, and the rel- 
ative difference in the fracture toughness between the cohesive failure of adhesives and the interface failure.

In the range of $A=0-0.19$, the fracture mode, which is basically interfacial, is accompanied by the formation of cracks in the cohesive zone or microscopic crack creation involving multiple concavoconvex microstructures ahead of the main crack tip. In this fracture mode, energy is consumed independent of the microscopic crack-tip position within the concavo-convex microstructure during crack propagation; thus, $G_{\text {IA }}$, which is the average energy consumption per unit crack area, is expressed as shown by Equation (5):

$$
G_{\mathrm{IA}}=\frac{w G_{\mathrm{IC}}+2 h G_{\mathrm{IIC}}}{w}=G_{\mathrm{IC}}+2 G_{\mathrm{IIC}} A
$$

where $G_{\text {IC }}$ and $G_{\text {IIC }}$ represent the pure mode I and mode II fracture toughness, respectively. This equation indicates that the increase in $G_{\mathrm{IA}}$ is caused by two factors: the presence of higher mode II fracture toughness $\left(G_{\text {IIC }}\right)$ and an increase in the actual crack length or the aspect ratio $(A)$. Thus, $G_{\mathrm{IA}}$ increases in proportion to the increase in the aspect ratio $A$, where the proportionality factor corresponds to $2 G_{\text {IIC. }}$.

Figure 14 shows the experimentally obtained $G_{\mathrm{IA}}$ and estimated $G_{\mathrm{IA}}$ calculated by Equation (5). The values of $G_{\mathrm{IC}}$ and $G_{\mathrm{IIC}}$ in Equation (5) are 179.6 \pm 28.17 and $513.8 \pm 69.06 \mathrm{~J} / \mathrm{m}^{2}$, respectively, which were obtained by preliminary $\mathrm{DCB}$ and end-notched-fracture (ENF) tests of the flat interface $(A=0)$ specimens. The ENF tests were conducted by following JIS K 7086 [21]. The crack propagation in a single ENF test is unstable; thus, the span length was changed

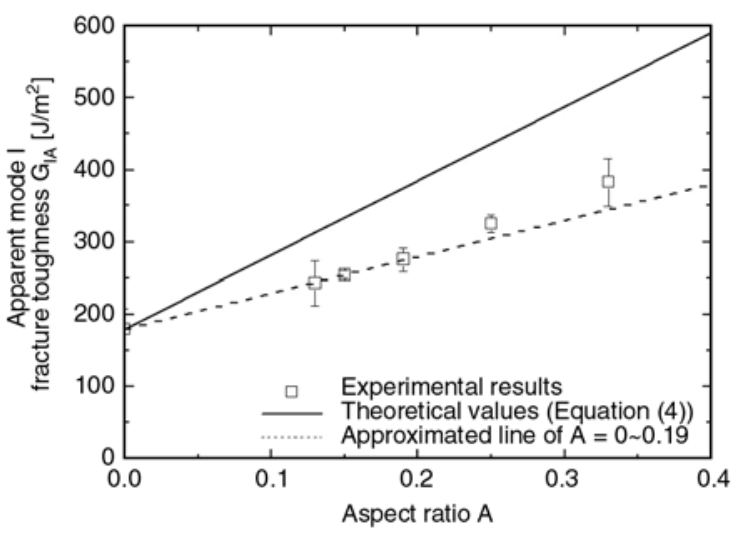

Figure 14. Comparison of estimated and experimental results of the apparent mode I fracture toughness $G_{\text {IA }}$ as a function of aspect ratio after unloading and the ENF tests were repeated to obtain mode II $R$ curves.

The dashed line in Figure 4 indicates the approximated line of experimental $G_{\mathrm{IA}}$ values of the interfacial fracture cases from $A=0$ to 0.19 . In the range from $A=0$ to 0.19 , the experimental results confirm a linear increase as $\mathrm{A}$ increases, as estimated in Equation (5), although the proportionality factor in the experimental results is lower than that of the estimation. This may be because Equation (5) does not take into account the effect of the stress concentration at the corner of the microstructure. The presence of this stress concentration enhances crack propagation, thus lowering the fracture toughness in the experiments compared to the value estimated in Equation (5).

Figure 14 also shows that the $G_{\text {IA }}$ values of the cohesive failure modes of $A=0.25$ and 0.33 are somewhat higher than the approximated line of the interfacial fracture mode. The higher $G_{\mathrm{IA}}$ is attributed to the higher energy dissipation due to the presence of cohesive failure, in addition to the complete interfacial failure of the microscopic regions of mode I and II. It is expected that in the case of cohesive failure accompanied by the partial interfacial failure of only the microscopic mode I region (such as in the case of a high aspect ratio, as shown in Figure 1), the energy required is less than needed for interfacial failure; thus, $G_{\text {IA }}$ may be lower than the approximated dashed line. However, because the crack propagation was determined by a microscopic stress field and not by total energy dissipation, the cohesive failure mode, above the dashed line, occurred. In future research, a crack-propagation simulation using the finite-element method and a quantitative investigation of the fracture mode transition should be done, as well as investigations of the mode II and mixed-mode loading conditions, and the influence of thermal residual stresses on the fracture behavior.

\section{Conclusions}

In-mold surface preparation using imprint lithography was proposed for polymer composite materials and applied to the adhesive joints of CFRPs. Because this method allows surface treatments to be done as composite materials are forming, it reduces time and costs compared to those required in conventional techniques. Concavo-convex microstructures fabricated on the surface of CFRPs enhanced the 
resistance to mode I crack propagation at the CFRP/ adhesive interface and induced microscopic mode II interfacial failure and cohesive failure, which require high energies for crack propagation. DCB tests for assessing mode I fracture toughness confirmed that the steady-state apparent fracture toughness $G_{\mathrm{IA}}$ became higher as the aspect ratio of the concavoconvex microstructures increased, and was 113\% higher than that without in-mold surface preparation, which was nearly the same as that achieved by sanding treatment. Thus, replacing the conventional treatments with the proposed method can eliminate the need for adverse secondary processes, enabling fast and cost-effective mass-production. Observations of interfaces during DCB tests revealed that the improvement of $G_{\text {IA }}$ was caused by the increase in the microscopic actual crack length and microscopic mode II fracture area per unit length on the lateral side of the concavo-convex microstructure, as well as a fracture-mode transition from interfacial to cohesive failure.

\section{References}

[1] Suzuki T., Takahashi J.: LCA of lightweight vehicles by using CFRP for mass produced vehicles. in 'Proceedings of $15^{\text {th }}$ International Conference on Composite Materials, Durban, South Africa' p.4 (2005).

[2] Yamasaki M., Taketa I., Yamaguchi K., Wadahara E., Kamae T., Iwasawa S., Sekido T., Kitano A.: ALSTECC program: Characterization of a short cycle RTM for mass production. in 'Proceedings of $16^{\text {th }}$ International Conference on Composite Materials, Kyoto, Japan' p.7 (2007).

[3] Boatman E. S., Covert D., Kalman D., Luchtel D., Omenn G. S.: Physical, morphological, and chemical studies of dusts derived from the machining of composite-epoxy materials. Environmental Research, 45, 242-255 (1988).

DOI: $10.1016 /$ S0013-9351(88)80050-1

[4] Matsuzaki R., Suzuki T.: Surface modification by nanoimprint lithography for improvement of the joint strength of composites. Journal of Solid Mechanics and Materials Engineering, 4, 963-973 (2010). DOI: $10.1299 /$ jmmp.4.963

[5] Chou S., Krauss P., Renstrom P.: Imprint of sub-25 nm vias and trenches in polymers. Applied Physics Letters, 67, 3114-3116 (1995).

DOI: $10.1063 / 1.114851$

[6] Chou S. Y., Krauss P. R., Renstrom P. J.: Imprint lithography with 25-nanometer resolution. Science, 272, 85-87 (1996).

DOI: $10.1126 /$ science. 272.5258 .85
[7] Schmitt H., Amon B., Beuer S., Petersen S., Rommel M., Bauer A. J., Ryssel H.: UV nanoimprint lithography process optimization for electron device manufacturing on nanosized scale. Microelectronic Engineering, 86, 636-638 (2009).

DOI: $10.1016 /$ j.mee.2008.11.017

[8] Abad E., Merino S., Retolaza A., Juarros A.: Design and fabrication using nanoimprint lithography of a nanofluidic device for DNA stretching applications. Microelectronic Engineering, 85, 818-821 (2008). DOI: 10.1016/j.mee.2007.12.048

[9] Yang R., Lu B-R., Xue J., Shen Z-K., Xu Z-C., Huq E., Qu X-P., Chen Y., Liu R.: Nanoimprint lithography for optic fluidics with phase gratings for environmental monitoring application. Microelectronic Engineering, 87, 824-826 (2010).

DOI: $10.1016 /$ j.mee.2009.11.030

[10] Kim J., Kim H. S., Lee D. G.: Investigation of optimal surface treatments for carbon/epoxy composite adhesive joints. Journal of Adhesion Science and Technology, 17, 329-352 (2003). DOI: $10.1163 / 156856103762864651$

[11] Shahid M., Hashim S.: Effect of surface roughness on the strength of cleavage joints. International Journal of Adhesion and Adhesives, 22, 235-244 (2002).

DOI: $10.1016 / \mathrm{S} 0143-7496(01) 00059-8$

[12] Cho T. M., Choo Y. S., Lee M. J., Oh H. C., Lee B. C., Park T. H., Shin Y. S.: Effect of surface roughness on the adhesive strength of the heat-resistant adhesive RTV88. Journal of Adhesion Science and Technology, 23, 1875-1882 (2009).

DOI: $10.1163 / 016942409 X 12508517390671$

[13] Prolongo S., Rosario G., Ureña A.: Study of the effect of substrate roughness on adhesive joints by SEM image analysis. Journal of Adhesion Science and Technology, 20, 457-470 (2006).

DOI: $10.1163 / 156856106777144345$

[14] Zhang S., Panat R., Hsia K.: Influence of surface morphology on the adhesion strength of epoxy-aluminum interfaces. Journal of Adhesion Science and Technology, 17, 1685-1711 (2003).

DOI: $10.1163 / 156856103322396749$

[15] Zhang Y., Spinks G. M.: An atomic force microscopy study of the effect of surface roughness on the fracture energy of adhesively bonded aluminium. Journal of Adhesion Science and Technology, 11, 207-223 (1997). DOI: 10.1163/156856197X00318

[16] Kim W-S., Yun I-H., Lee J-J., Jung H-T.: Evaluation of mechanical interlock effect on adhesion strength of polymer-metal interfaces using micro-patterned surface topography. International Journal of Adhesion and Adhesive, 30, 408-417 (2010).

DOI: $10.1016 /$ j.ijadhadh.2010.05.004

[17] Janarthanan V., Garrett P. D., Stein R. S., Srinivasarao M.: Adhesion enhancement in immiscible polymer bilayer using oriented macroscopic roughness. Polymer, 38, 105-111 (1997).

DOI: $\underline{10.1016 / \mathrm{S} 0032-3861(96) 00447-8}$ 
[18] Rider A. N., Arnott D. R.: The influence of adherend topography on the fracture toughness of aluminiumepoxy adhesive joints in humid environments. The Journal of Adhesion, 75, 203-228 (2001).

DOI: $10.1080 / 00218460108029601$

[19] Zavattieri P. D., Hector L. G., Bower A. F.: Determination of the effective mode-I toughness of a sinusoidal interface between two elastic solids. International Journal of Fracture, 145, 167-180 (2007).

DOI: $10.1007 / \mathrm{s} 10704-007-9109-\mathrm{y}$

[20] Volinsky A. A., Moody N. R., Gerberich W. W.: Interfacial toughness measurements for thin films on substrates. Acta Materialia, 50, 441-466 (2002). DOI: 10.1016/S1359-6454(01)00354-8

[21] JIS K 7086: Testing methods for interlaminar fracture toughness of carbon fibre reinforced plastics (2011).
[22] Blackman B. R. K., Kinloch A. J., Paraschi M., Teo W. S.: Measuring the mode I adhesive fracture energy, $G_{\text {IC }}$, of structural adhesive joints: The results of an international round-robin. International Journal of Adhesion and Adhesives, 23, 293-305 (2003). DOI: 10.1016/S0143-7496(03)00047-2

[23] Evans A. G., Hutchinson J. W.: Effects of non-planarity on the mixed mode fracture resistance of bimaterial interfaces. Acta Metallurgica, 37, 909-916 (1989).

DOI: 10.1016/0001-6160(89)90017-5

[24] Bradley W., Cohen R.: Matrix deformation and fracture in graphite-reinforced epoxies. in 'Delamination and debonding of materials' (ed.: Johnson W. S.) ASTM, Philadephia, 389-410 (1985).

[25] Miyairi H.: Basis and application of structural adhesion. CMC Publishing, Tokyo (2006). 